\title{
EFFECT OF PHYTASE ENZYME AND CITRIC ACID ON PRODUCTIVE PERFORMANCE, NUTRIENT RETENTION AND TIBIA BONE OF BROILER CHICKS FED LOW AVAILABLE PHOSPHORUS DIET
}

\author{
Naglaa K. Soliman ${ }^{1}$ and Y.M. Al- Youssef ${ }^{2}$ \\ ${ }^{1}$ Poult. Prod. Dep., Fac. of Agric., Ain Shams Univ., Cairo 11241, Egypt. \\ ${ }^{2}$ Anim. Prod. Sci. and Fish Dep. College of Agric. and Food Sci. King Faisal Uni., Saudi Arabia.
}

(Received 15/10/2020, accepted 14/11/2020)

\section{SUMMARY}

\begin{abstract}
$\mathrm{T}$ The study investigate the effect of feeding low available phosphorus (AP) diet supplemented with three levels of microbial phytase (MP) and /or three levels of citric acid (CA) on broiler performance, tibia bone and nutrient retention. Four hundred day- old Ross broiler chicks were randomly allocated into ten treated groups, with four replicates of 10 birds each. The chicks were fed ten different diets as normal phosphorus $0.45 \%$ AP (positive control, PC) and nine groups with phosphorus deficient diets $0.25 \%$ AP (negative control, NC) supplemented with three CA levels as $0 \%, 1 \%$ or $2 \%$ and / or MP levels 0,500 and $1000 \mathrm{FTU} / \mathrm{Kg}$. The results indicated that supplementation of MP and /or CA into NC diet significantly improved body gain of chicks. Feed intake significantly increased due to adding $1 \%$ CA and MP levels into NC diet. Feed conversion ratio improved due to supplementing MP or CA into NC diet. There is no significant effect on tibia width and breaking strength by adding MP and/or CA into NC diet. Adding MP into NC diet significantly improved tibia length, weight, and ash content while, citric acid affected significantly at the first stage of growth only. Blood phosphorus increased significantly by supplementing MP into NC diet at the $5^{\text {th }}$ week of age, while blood calcium was not affected significantly by MP and/or CA. Tibia calcium of chicks fed NC diet was higher than those fed PC diet or NC diet enriched with MP and/or CA. Tibia phosphorus did not response clearly for adding MP or CA into NC diet. Nitrogen and calcium retention of chicks fed NC were significantly lower than those fed PC or NC diet supplemented with 1000 FTU/kg MP and CA. Phosphorus retention of chicks fed NC diet was significantly similar for those fed PC or NC diets supplemented with MP and/or CA. It can be concluded that adding MP at $500 \mathrm{FTU} / \mathrm{kg}$ level with $1 \% \mathrm{CA}$ into NC diet was effectiveness to improve body weight gain and tibia bone characteristics as like as those fed PC or NC diet enriched with 1000 FTU/kg MP.
\end{abstract}

Keywords: Broiler performance, phosphorus level, blood analysis, bone measurements and nutrient utilization.

\section{INTRODUCTION}

Corn and soy are commonly used in broiler diets where two thirds of phosphorus content is in the form of phytate which is unavailable for poultry due to insufficient endogenous phytase enzyme (Woyengo et al. 2010; Deepa et al., 2011). Phosphorus represents an essential element for growth and bone formation of broiler chicks. As well, phosphorus is considered one of the most expensive feed ingredients in broiler diets (Ismail et al., 2011). Therefore, high percentage of unavailable plant phosphorus represents an economic problem due to addition of inorganic expensive phosphorus sources which increase the diets cost. Furthermore, excretion of unavailable phosphorus can result in environmental pollution Vieira et al., (2018).

Supplementation of phytase into broiler diets established as a mean to increase plant phosphorus utilization, retention and to reduce the addition of expensive inorganic phosphorus in broiler diets (Cowieson et al., 2006). As well, addition of phytase improves the efficiency of dietary protein and energy utilization and increases the performance characteristics of broiler chicks (Deepa et al., 2011).

Intestinal $\mathrm{pH}$ is one of the several factors affecting the efficacy of exogenous phytase enzyme, which is most active at 2.5 to5.5 pH (Abd El-Hack et al., 2018). Therefore, the effectiveness of phytase may be enhanced by reducing intestinal $\mathrm{pH}$ with supplementation of organic acids (Nourmohammadi et al., 2010) such as citric acid (El-Afifi et al., 2001) which has been reported to improve the efficacy of phytase (Redcliffe et al., 1998). Several studies indicated that citric acid has been used successfully as 
feed additives into broiler diets to improve the digestibility and retention of dietary phosphorus, calcium and protein, due to its role in increasing the solubility of minerals and intestinal villi length, (El-Afifi et al., 2001, Aydin et al., 2010 and Soliman et al., 2012). Furthermore, addition of citric acid into phosphorus deficient diets increase phytate-phosphorus utilization (Boling et al., 2000) and enhances the efficiency of phytase (Redcliffe et al., 1998). Previous studies (Brenes et al., 2003; Hariharan \& Gangadevi 2015) noted an interaction between citric acid and phytase enzyme in improving performance and mineral retention of broiler chicks. Therefore, citric acid may be useful to improve the efficacy of phytase enzyme and reduce the level of its supplementation into broiler diets. The current study aimed to determine the effect of adding citric acid and/or phytase enzyme into low available phosphorus (AP) diet on performance of broiler chicks. As well, the complimentary effect on tibia bone, minerals and nitrogen retention were included.

\section{MATERIALS AND METHODS}

\section{Experimental Procedure:}

The experimental procedure was approved by the Institutional Animal and Poultry care and use committee at College of Agriculture Sciences and Food King Faisal University Saudi Arabia Kingdom. Four-hundred-day-old Ross 308 broiler chicks were allocated randomly into 10 treated groups with four replicates of 10 birds each. Two diets (Table 1) were formulated according to NRC (1994) as normal phosphorus diets $0.45 \%$ AP (positive control PC) and phosphorus deficient diets $0.25 \%$ AP (negative control NC) which was supplemented with three levels of microbial phytase (MP), three levels of citric acid (CA) and three levels of their combination. The experimental diets were T1, normal phosphorus $\operatorname{diet}(\mathrm{PC})$; T2, phosphorus deficient diet (NC); T3, NC diet + 1\% CA; T4, NC diet + 2\% CA; T5, NC diet + 500 FTU MP; T6, NC diet +500 FTU MP+ 1\% CA; T7, NC diet + 500 FTU MP + 2\% CA; T8, NC diet+ 1000 FTU MP; T9, NC diet + 1000 FTU MP + 1\% CA; T10, NC diet +1000 FTU MP + 2\% CA.

The 35 days experimental period was divided into two stages, from 0 to 3 weeks and from 3 to 5 weeks of age. Water and mash feed were provided $a d$ lib. The chicks were placed in floor pens with wood-shavings litter. Fan and air conditions were used for keeping suitable temperature. Artificial lighting was provided constantly.

Body weights were recorded weekly for each chick and average body weight gain was calculated for each replicate and treatment group. Feed intakes were obtained weekly in gram, and feed conversion ratio was calculated as gram feed /gram gain.

At the end of the two experimental stages 8 chicks per treatment representing the four replicate groups were slaughtered. For separating serum blood samples were collected and centrifuged at 1500 rpm for 10 minutes. Serum was decanted and frozen till chemical analysis. Plasma phosphorus and calcium concentrations were determined using special chemical kits from United Diagnostic Industry, Dammam, Saudi Arabia Kingdom.

Slaughtered birds were defeathered, eviscerated and the internal organs were separated. Right and left tibia were excised, defleshed and dried at $105^{\circ} \mathrm{C}$. Right tibia was weighted in grams and its width, length was measured by caliper in mm (Zhang \& Coon 1997). Tibia bone was ashed in muffle furnace at $600^{\circ} \mathrm{C}$ and ash weight was calculated as percentage of dry tibia (Chung \& Baker 1990). Tibia content of calcium and phosphorus were determined according to (AOAC 1990). Left tibia was used to measure tibia breaking strength (TBS) in $\mathrm{kg} / \mathrm{cm}^{2}$. TBS was determined by applying the simple three-point bending concept using Instron Universal Testing Machine. Determination process was conducted at the Research Center of Properties and Testing of Materials, Faculty of Engineering, Ain Shams University. TBS was defined as the upper limit force that the tibia bone can afford when applied perpendicularly on the longitudinal axis of this bone and before the bone itself is broken (Crenshaw et al., 1981).

\section{Digestibility Trail and Chemical Assays:}

Digestibility trail was conducted at the end of experiment using four chicks from each treatment represented the four replicates group. The chicks were housed in individual cages with trays for collecting excreta and calculating feed intake for three days. Excreta was homogenized, weighed, and dried in an oven at $60 \mathrm{C}^{\circ}$ then representative samples were taken for determining nitrogen, calcium and phosphorus content. Nitrogen was determined using Kjeldahl method AOAC (1990), and apparent nitrogen retention was calculated as: $\mathrm{N}$ intake $-\mathrm{N}$ output/ $\mathrm{N}$ intake $\times 100$. For calculating phosphorus and calcium retention, excreta samples were ashed in muffle furnace at $600^{\circ} \mathrm{C}$ then digested with nitric 
perchloric. Calcium and phosphorus content of excreta were determined using spectrophotometer and special chemical kits (United Diagnostic industry, Dammam, Saudi Arabia kingdom). Mineral retention percentage was calculated as: $\mathrm{Ca}$ or $\mathrm{P}$ intake $-\mathrm{Ca}$ or $\mathrm{P}$ output / Ca or P intake $\times 100$.

Table (1): Composition and calculated analysis of experimental basal control diets.

\begin{tabular}{|c|c|c|}
\hline Item & $\begin{array}{c}\text { Positive control diet } \\
0.45 \% \text { A.P }\end{array}$ & $\begin{array}{c}\text { Negative control diet } \\
0.25 \% \text { A.P }\end{array}$ \\
\hline \multicolumn{3}{|l|}{ Ingredients \%: } \\
\hline Yellow corn & 55.15 & 57.19 \\
\hline Soybean meal (48\%) & 37.3 & 37 \\
\hline Vegetable oil & 3.7 & 3 \\
\hline Dicalcium phosphate & 1.7 & 0.66 \\
\hline Limestone & 1.5 & 1.5 \\
\hline Common salt & 0.25 & 0.25 \\
\hline Vit. \& min. premix* & 0.2 & 0.2 \\
\hline DL. Methionine & 0.2 & 0.2 \\
\hline Total & 100 & 100 \\
\hline \multicolumn{3}{|l|}{ Calculated composition: } \\
\hline Crude protein $\%$ & 22.6 & 22.6 \\
\hline M.E. Kcal/kg & 3107 & 3107 \\
\hline$\%$ Calcium & 1.05 & 0.825 \\
\hline \% Total phosphorus & 0.703 & 0.511 \\
\hline \%Available phosphorus & 0.443 & 0.25 \\
\hline \% Methionine + cysteine & 0.916 & 0.92 \\
\hline \%Lysine & 1.24 & 1.24 \\
\hline
\end{tabular}

\section{Statistical Analysis:}

Data were subjected to analysis of variance using general linear model (GLM) procedure described in SAS User's Guide 1998. Differences among means were tested using Duncan's multiple range tests. The following two models were used for data analysis: Model 1, to compare among the ten treated groups including positive control while model 2 was used to determine the main effects (phytase, citric) and their interaction.

1. $\mathrm{Y}_{\mathrm{ij}}=\mu+\mathrm{MPCA}_{\mathrm{i}}+\mathrm{e}_{\mathrm{ij}}$

2. $\mathrm{Y}_{\mathrm{ijk}}=\mu+\mathrm{MP}_{\mathrm{i}}+\mathrm{CA}_{\mathrm{j}}+(\mathrm{MP} \times \mathrm{CA})_{\mathrm{ij}}+\mathrm{e}_{\mathrm{ijk}}$

Where $\mathrm{Y}_{\mathrm{ijk}}, \mathrm{Y}_{\mathrm{ij}}=$ individual observation, $\mu=$ experimental mean, $\mathrm{MP}_{\mathrm{i}}=$ microbial phytase effect, $\mathrm{CA}_{\mathrm{j}}=$ citric effect, $(\mathrm{MP} \times \mathrm{CA})_{\mathrm{ij}}=$ the interaction between phytase and citric, $\mathrm{MPCA}_{\mathrm{i}}=$ effect of phytase and citric, $\mathrm{e}_{\mathrm{ijk}}, \mathrm{e}_{\mathrm{ij}}=$ experimental error.

\section{RESULTS AND DISCUSSION}

\section{Performance Measurements:}

Performance aspects of chicks are shown in Table 2. It is clear that the chicks fed NC diet recorded the lowest body weight gain value. However, the overall mean of mortality (data not shown) was less than $2 \%$ and not related to feeding phosphorus deficient diets. Supplementation of MP or/ and CA into $\mathrm{NC}$ diet significantly $(\mathrm{P} \leq 0.05)$ improved body gain of chicks. Several studies proved that, addition of phytase into low AP diets significantly improved body weight of broiler chicks (Brenes et al., 2003; Ahmad et al. 2000). The mode of action of phytase in improving body weight gain may be due to release of $\mathrm{P}$ from phytate, which enhanced the digestibility of starch and energy production 
(Ebrahimnezhad et al. 2008). The improvement effect of CA on body weight gain have been reported previously and related to its effect in reducing digestive tract $\mathrm{pH}$. Low $\mathrm{pH}$ value is responsible for enhancing nutrients utilization and hence improves body weight gain (El-Afifi 2003; Talebi et al., 2010). Body gains of birds fed $500 \mathrm{FTU} / \mathrm{Kg}$ MP with 1 or $2 \%$ of CA (T6 \& T7) were significantly higher than those fed PC (T1) diet at 5 weeks of age and similar at 3 weeks of age. As well as the interaction between phytase and citric acid for improving body gain was significant $(\mathrm{P} \leq 0.05)$ at the second stage of growth. The current result indicate to complimentary effect between MP and CA in improving body weight gain and the level of $500 \mathrm{FTU} / \mathrm{Kg}$ MP with 1 or $2 \% \mathrm{CA}$ is effectiveness

Table (2): Effect of phytase and/or citric acid supplementation on performance of broiler chicks.

\begin{tabular}{|c|c|c|c|c|c|c|c|c|}
\hline \multicolumn{3}{|l|}{ Treatments } & \multicolumn{2}{|c|}{ Body weight gain (g) } & \multicolumn{2}{|c|}{ Feed intake $(\mathrm{g})$} & \multirow{2}{*}{$\begin{array}{l}\text { Feed } \\
\qquad 0-3 \text { wk }\end{array}$} & \multirow{2}{*}{$\begin{array}{r}\text { conversion } \\
\text { ratio } \\
0-5 \text { wk }\end{array}$} \\
\hline A.P \% & $\begin{array}{r}\text { MPFTU } \\
/ \mathrm{kg}\end{array}$ & $\begin{array}{r}\mathrm{CA} \\
\%\end{array}$ & $0-3$ wk & $0-5 \mathrm{wk}$ & $0-3$ wk & $0-5 \mathrm{wk}$ & & \\
\hline $\mathrm{T} 1,0.45(\mathrm{PC})$ & - & - & $594.6^{\mathrm{ab}}$ & $1784.0^{\mathrm{bcd}}$ & $829.1^{\text {bc }}$ & $3202.9^{\text {cde }}$ & $1.39^{\mathrm{b}}$ & $1.79^{\mathrm{abc}}$ \\
\hline $\mathrm{T} 2,0.25(\mathrm{NC})$ & 0 & 0 & $344.0^{\mathrm{e}}$ & $1106.4^{\mathrm{e}}$ & $573.7^{\mathrm{d}}$ & $2139.9^{f}$ & $1.68^{\mathrm{ab}}$ & $1.95^{\mathrm{ab}}$ \\
\hline $\mathrm{T} 3,0.25$ & 0 & $1 \%$ & $490.2^{\mathrm{cd}}$ & $1578.8^{\mathrm{d}}$ & $866.9^{\mathrm{abc}}$ & $3035.3^{\mathrm{de}}$ & $1.83^{\mathrm{a}}$ & $1.96^{\mathrm{a}}$ \\
\hline $\mathrm{T} 4,0.25$ & 0 & $2 \%$ & $455.8^{d}$ & $1744.7^{\mathrm{cd}}$ & $758.3^{\mathrm{c}}$ & $2952.0^{\mathrm{e}}$ & $1.66^{\mathrm{ab}}$ & $1.69^{c}$ \\
\hline $\mathrm{T} 5,0.25$ & 500 & 0 & $563.7^{\mathrm{bc}}$ & $1757.0^{\mathrm{cd}}$ & $827.5^{\mathrm{bc}}$ & $3273.5^{\text {bcde }}$ & $1.47^{\mathrm{b}}$ & $1.86^{\mathrm{abc}}$ \\
\hline $\mathrm{T} 6,0.25$ & 500 & $1 \%$ & $588.6^{\mathrm{abc}}$ & $2083.7^{\mathrm{a}}$ & $901.4^{\mathrm{ab}}$ & $3669.4^{\mathrm{a}}$ & $1.54^{\mathrm{ab}}$ & $1.76^{\mathrm{abc}}$ \\
\hline $\mathrm{T} 7,0.25$ & 500 & $2 \%$ & $574.0^{\mathrm{bc}}$ & $1906.9^{\mathrm{abc}}$ & $872.0^{\mathrm{abc}}$ & $3289.2^{\mathrm{bcd}}$ & $1.52^{\mathrm{ab}}$ & $1.73^{\mathrm{bc}}$ \\
\hline $\mathrm{T} 8,0.25$ & 1000 & 0 & $607.3^{\mathrm{ab}}$ & $2076.3^{\mathrm{a}}$ & $907.2^{\mathrm{ab}}$ & $3587.4^{\mathrm{ab}}$ & $1.50^{\mathrm{ab}}$ & $1.73^{\mathrm{bc}}$ \\
\hline $\mathrm{T} 9,0.25$ & 1000 & $1 \%$ & $5^{\mathrm{a}} 675$ & $2036.0^{\mathrm{ab}}$ & $977.4^{\mathrm{a}}$ & $3699.1^{\mathrm{a}}$ & $1.45^{\mathrm{b}}$ & $1.82^{\mathrm{abc}}$ \\
\hline \multirow{2}{*}{$\mathrm{T} 10,0.25$} & 1000 & $2 \%$ & $534.0^{\text {bcd }}$ & $1977.2^{\mathrm{abc}}$ & $847.6^{\mathrm{bc}}$ & $3411.0^{\mathrm{abc}}$ & $1.59^{\mathrm{ab}}$ & $1.72^{\mathrm{bc}}$ \\
\hline & & SEM & 31.1 & 80.5 & 34.6 & 102.9 & 0.10 & 0.069 \\
\hline \multicolumn{9}{|c|}{ Overall Means Phytase } \\
\hline 0.25 & 0 & - & $430.0^{\mathrm{b}}$ & $1476.6^{\mathrm{b}}$ & $733.0^{\mathrm{b}}$ & $2709.1^{b}$ & $1.73^{\mathrm{a}}$ & 1.87 \\
\hline 0.25 & 500 & - & $575.4^{\mathrm{a}}$ & $1915.9^{\mathrm{a}}$ & $867.0^{\mathrm{a}}$ & $3410.7^{\mathrm{a}}$ & $1.51^{\mathrm{b}}$ & 1.79 \\
\hline 0.25 & 1000 & - & $605.6^{\mathrm{a}}$ & $2029.8^{\mathrm{a}}$ & $910.7^{\mathrm{a}}$ & $3565.8^{\mathrm{a}}$ & $1.51^{\mathrm{b}}$ & 1.76 \\
\hline \multicolumn{9}{|c|}{ Overall Means Citric } \\
\hline 0.25 & - & 0 & $505.0^{\mathrm{b}}$ & $1646.6^{\mathrm{b}}$ & $769.5^{\mathrm{b}}$ & $3000.2^{\mathrm{c}}$ & 1.55 & $1.85^{\mathrm{a}}$ \\
\hline 0.25 & - & $1 \%$ & $584.8^{\mathrm{a}}$ & $1899.5^{\mathrm{a}}$ & $915.2^{\mathrm{a}}$ & $3468.0^{\mathrm{a}}$ & 1.61 & $1.85^{\mathrm{a}}$ \\
\hline 0.25 & - & $2 \%$ & $521.3^{\mathrm{b}}$ & $1876.3^{\mathrm{a}}$ & $826.0^{\mathrm{b}}$ & $3217.4^{\mathrm{b}}$ & 1.59 & $1.71^{\mathrm{b}}$ \\
\hline \multicolumn{3}{|c|}{ Interaction MP x CA } & NS & 0.004 & 0.01 & 0.002 & NS & NS \\
\hline
\end{tabular}

A.V, available phosphorus; $M P$, microbial phytase; CA, citric acid; PC, positive control (0.45\% phosphorus); NC, negative control $(0.25 \%$ phosphorus)

$a, b, c, d$ Mean values within a column with no common superscript differ significantly from each other (P<0.05). SEM, standard error of means; NS, not significant

The value \pm stander error

to improve body gain of chicks fed phosphorus deficient diet as like as those fed PC diet (T1) or 1000 FTU/Kg MP (T8). The present finding confirms the results of Nourmohammadi et al. (2012) noted extra increment in body weight gain of broiler chicks fed diets enriched with phytase and citric acid. This increment is related to increase phytase efficiency as results of low intestinal $\mathrm{pH}$ induced by citric acid (Brenes et al., 2003 \& Vieira et al., 2018).

The overall values of feed intake significantly increased $(\mathrm{P} \leq 0.05)$ due to adding $1 \% \mathrm{CA}$ and MP into diets compared with $2 \% \mathrm{CA}$ or un-supplemented groups. As well, the interaction between CA and MP in increasing feed intake was significant at both stages of growth. The reduction in feed consumption of chicks fed low AP diets was observed by Sacakli et al. (2006). As well, inclusion CA with $2 \%$ may reduce feed intake due to its effect in lowering palatability and increasing the transmission time of feed in intestinal tract (Nourmohammadi et al., 2010). Feed conversion ratio improved due to adding MP or $\mathrm{CA}$ into $\mathrm{NC}$ diet, the improvement was significant $(\mathrm{P} \leq 0.05)$ at the $3^{\text {rd }}$ and $5^{\text {th }}$ week for MP and CA respectively.

The improvement in feed conversion can be related to increase protein digestibility and retention reported in the current study (Table 5). The results are in harmony with those of Zobac et al. (2004) and El-Afifi (2003) who observed an improvement in feed conversion ratio due to adding phytase or CA respectively into broiler diets. 


\section{Tibia Ash and Morphometry:}

The values of tibia width and breaking strength (Table 3) were not differed significantly due to adding MP and/or CA into NC diet as well, the interaction lacked significance. These results are consistence with Sreeja et al. (2018) who did not observe any differences in tibia width by adding phytase into low AP diets.

Table (3): Effect of phytase and/or citric acid supplementation on tibia morphometry and ash content.

\begin{tabular}{|c|c|c|c|c|c|c|c|c|c|c|c|c|c|c|}
\hline \multicolumn{3}{|l|}{ Treatments } & \multicolumn{2}{|c|}{$\begin{array}{r}\text { Tibia width } \\
(\mathrm{mm})\end{array}$} & \multicolumn{2}{|c|}{$\begin{array}{l}\text { Tibia length } \\
\qquad(\mathrm{mm})\end{array}$} & \multicolumn{2}{|c|}{$\begin{array}{l}\text { Tibia weight } \\
\text { (g) }\end{array}$} & \multicolumn{2}{|c|}{ Tibia ash (g) } & \multicolumn{2}{|c|}{ Tibia Ash (\%) } & \multicolumn{2}{|c|}{$\begin{array}{r}\text { Tibia strength } \\
\mathrm{Kg} / \mathrm{cm}^{2}\end{array}$} \\
\hline \multirow[t]{2}{*}{ A.P \% } & & $\mathrm{CA}$ & $0-3$ & $0-5$ & $0-3$ & $0-5$ & $0-3$ & $0-5$ & $0-3$ & $0-5$ & $0-3$ & $0-5$ & $0-3$ & $0-5$ \\
\hline & $\begin{array}{r}\text { FTU/ } \\
\mathrm{kg}\end{array}$ & $\%$ & Wk & Wk & Wk & wk & wk & Wk & wk & wk & wk & wk & wk & wk \\
\hline $\mathrm{T} 1,0.45$ (PC) & - & - & 4.88 & $7.34^{\mathrm{a}}$ & $63.69^{\mathrm{abc}}$ & $84.83^{b c}$ & $2.00^{\mathrm{bc}}$ & $6.20^{\mathrm{ab}}$ & $0.68^{\mathrm{bc}}$ & $2.02^{\mathrm{ab}}$ & $13.90^{\mathrm{bc}}$ & $32.66^{\mathrm{ab}}$ & 14.67 & 23.50 \\
\hline $\mathrm{T} 2,0.25(\mathrm{NC})$ & 0 & 0 & 4.59 & $6.30^{\mathrm{b}}$ & $59.83^{\mathrm{e}}$ & $86.13^{\mathrm{abc}}$ & $1.9 b^{c}$ & $5.54^{\mathrm{ab}}$ & $0.57^{\mathrm{c}}$ & $1.81^{\mathrm{abc}}$ & $12.26^{\mathrm{cd}}$ & $32.73^{\mathrm{ab}}$ & 13.33 & 18.50 \\
\hline $\mathrm{T} 3,0.25$ & 0 & $1 \%$ & 4.63 & $5.91^{\mathrm{b}}$ & $63.86^{\mathrm{abc}}$ & $82.34^{\mathrm{c}}$ & $2.14^{\mathrm{abc}}$ & $4.85^{\mathrm{b}}$ & $0.64^{\mathrm{bc}}$ & $1.50^{\mathrm{c}}$ & $13.79^{\mathrm{bc}}$ & $30.98^{b}$ & 14.33 & 16.75 \\
\hline $\mathrm{T} 4,0.25$ & 0 & $2 \%$ & 5.31 & $6.43^{\mathrm{b}}$ & $60.09^{\mathrm{de}}$ & $86.29^{\mathrm{abc}}$ & $1.80^{\mathrm{c}}$ & $5.09^{\mathrm{ab}}$ & $0.56^{\mathrm{c}}$ & $1.80^{\mathrm{abc}}$ & $10.52^{\mathrm{d}}$ & $35.05^{\mathrm{a}}$ & 12.67 & 15.50 \\
\hline $\mathrm{T} 5,0.25$ & 500 & 0 & 4.86 & $6.46^{\mathrm{b}}$ & $62.11^{\text {bcde }}$ & $90.52^{\mathrm{ab}}$ & $2.13^{\mathrm{abc}}$ & $6.28^{\mathrm{ab}}$ & $0.75^{\mathrm{abc}}$ & $1.94^{\mathrm{abc}}$ & $15.48^{\mathrm{ab}}$ & $30.94^{b}$ & 12.00 & 22.25 \\
\hline T6, 0.25 & 500 & $1 \%$ & 5.18 & $6.05^{\mathrm{b}}$ & $63.09^{\mathrm{abcd}}$ & $89.85^{\mathrm{abc}}$ & $2.43^{\mathrm{ab}}$ & $5.84^{\mathrm{ab}}$ & $0.83^{\mathrm{ab}}$ & $2.01^{\mathrm{ab}}$ & $15.95^{\mathrm{ab}}$ & $34.42^{\mathrm{ab}}$ & 16.00 & 17.75 \\
\hline $\mathrm{T} 7,0.25$ & 500 & $2 \%$ & 5.07 & $5.98^{\mathrm{b}}$ & $64.8^{3 \mathrm{ab}}$ & $84.63^{b c}$ & $2.42^{\mathrm{ab}}$ & $4.92^{\mathrm{ab}}$ & $0.85^{\mathrm{ab}}$ & $1.62^{\mathrm{bc}}$ & $16.66^{\mathrm{ab}}$ & $32.94^{\mathrm{ab}}$ & 15.33 & 17.25 \\
\hline $\mathrm{T} 8,0.25$ & 1000 & 0 & 4.65 & $5.90^{\mathrm{b}}$ & $60.82^{\text {cde }}$ & $86.55^{\mathrm{abc}}$ & $1.95^{\mathrm{bc}}$ & $5.26^{\mathrm{ab}}$ & $0.64 b^{c}$ & $1.88^{\mathrm{abc}}$ & $13.68^{\mathrm{bc}}$ & $35.40^{\mathrm{a}}$ & 13.00 & 19.25 \\
\hline $\mathrm{T} 9,0.25$ & 1000 & $1 \%$ & 5.43 & $\begin{array}{r}6.56^{\mathrm{a}} \\
\mathrm{b}\end{array}$ & $65.92^{\mathrm{a}}$ & $89.81^{\mathrm{abc}}$ & $2.65^{\mathrm{a}}$ & $6.31^{\mathrm{ab}}$ & $0.93^{\mathrm{a}}$ & $2.20^{\mathrm{a}}$ & $17.17^{\mathrm{a}}$ & $34.98^{\mathrm{a}}$ & 17.33 & 21.25 \\
\hline \multirow[t]{2}{*}{$\mathrm{T} 10,0.25$} & 1000 & $2 \%$ & 4.64 & $6.30^{\mathrm{b}}$ & $63.48^{\mathrm{abcd}}$ & $93.46^{\mathrm{a}}$ & $1.95^{\mathrm{bc}}$ & $6.44^{\mathrm{a}}$ & $0.68^{\mathrm{bc}}$ & $2.11^{\mathrm{ab}}$ & $14.68^{\mathrm{abc}}$ & $33.06^{\mathrm{ab}}$ & 13.33 & 19.50 \\
\hline & & SEM & 2.66 & 0.29 & 1.09 & 2.38 & 0.18 & 0.44 & 0.07 & 0.15 & 0.97 & 1.20 & 1.79 & 2.39 \\
\hline \multicolumn{15}{|c|}{ Overall Means Phytase } \\
\hline 0.25 & 0 & - & 4.84 & 6.21 & $61.26^{\mathrm{b}}$ & $84.92 b$ & $1.95^{\mathrm{b}}$ & $5.16^{\mathrm{b}}$ & $0.59^{\mathrm{b}}$ & $1.70^{\mathrm{b}}$ & $12.19^{\mathrm{b}}$ & 32.92 & 13.44 & 16.92 \\
\hline 0.25 & 500 & - & 5.04 & 6.16 & $63.34^{\mathrm{a}}$ & $88.33^{\mathrm{ab}}$ & $2.33^{\mathrm{a}}$ & $5.68^{\mathrm{ab}}$ & $0.81^{\mathrm{a}}$ & $1.85^{\mathrm{ab}}$ & $16.03^{\mathrm{a}}$ & 32.76 & 14.44 & 19.08 \\
\hline 0.25 & 1000 & - & 4.91 & 6.25 & $63.41^{\mathrm{a}}$ & $89.94^{\mathrm{a}}$ & $2.18^{\mathrm{ab}}$ & $6.00^{\mathrm{a}}$ & $0.75^{\mathrm{a}}$ & $2.06^{\mathrm{a}}$ & $15.17^{\mathrm{a}}$ & 34.48 & 14.56 & 20.00 \\
\hline \multicolumn{15}{|c|}{ Overall Means Citric } \\
\hline 0.25 & - & 0 & 4.70 & 6.22 & $60.92^{\mathrm{a}}$ & 87.73 & $1.99^{\mathrm{b}}$ & 5.69 & $0.65^{\mathrm{b}}$ & 1.88 & $13.80^{\mathrm{b}}$ & 33.02 & 12.78 & 20.00 \\
\hline 0.25 & - & $1 \%$ & 5.08 & 6.17 & $64.29^{\mathrm{ab}}$ & 87.33 & $2.41^{\mathrm{a}}$ & 5.67 & $0.80^{\mathrm{a}}$ & 1.90 & $15.64^{\mathrm{a}}$ & 33.46 & 15.89 & 18.58 \\
\hline 0.25 & - & $2 \%$ & 5.01 & 6.24 & $62.80^{\mathrm{b}}$ & 88.12 & $2.06^{\mathrm{b}}$ & 5.48 & $0.70^{\mathrm{ab}}$ & 1.84 & $13.95^{\mathrm{ab}}$ & 33.68 & 13.78 & 17.42 \\
\hline Interact & on MP & $x$ CA & NS & NS & NS & NS & NS & NS & NS & NS & NS & NS & NS & NS \\
\hline
\end{tabular}

A.V, available phosphorus; MP, microbial phytase; CA, citric acid; PC, positive control (0.45\% phosphorus); NC, negative control ( $0.25 \%$ phosphorus)

$a, b, c, d$ Mean values within a column with no common superscript differ significantly from each other $(P<0.05)$. SEM, standard error of means; NS, not significant

The value \pm stander error

However, Zivkov et al. (2012) reported an improvement in tibia breaking strength due to supplement phytase into low AP broiler diets. This dissimilarity may be related to the differences in the experimental conditions, especially, level of phytase and method of measuring tibia strength. Tibia length, weight and ash content $(\mathrm{g})$ of chicks significantly $(\mathrm{P} \leq 0.05)$ improved due to adding MP into NC diet at both stages of growth, while citric acid affected significantly $(\mathrm{P} \leq 0.05)$ at the first stage of growth only. Brenes et al.(2003) and Sacakli et al.(2006) reported that tibia weight, length and ash content enhanced by supplying low AP broiler diets with citric acid or phytase due to its effect in increasing the retention rate of calcium and phosphorus. 


\section{Phosphorus and Calcium Values:}

Blood calcium levels were not affected significantly by adding MP and/or CA into NC diet at the two stages of growth (Table 4). The current finding agrees with Hariharan \& Gangadevi (2015) who did not observe any significant effect on plasma concentration of calcium due to adding phytase or citric acid into low phosphorus diets. On the other hand, Sheihk et al. (2010) stated that organic acid supplementation increased blood concentration of calcium. This disagreement may be related to use different levels and types of organic acids, butyric, fumaric and lactic in their experiment against citric acid in the current work. Blood phosphorus significantly $(\mathrm{P} \leq 0.05)$ increased due to adding MP into low AP diet at the $5^{\text {th }}$ week of age, while CA supplementation has no effect. The present results are in harmony with the finding of Luciana et al. (2016) that, phytase supplementation can increase blood concentration of phosphorus. In contrary Hariharan \& Gangadevi (2015) reported that blood phosphorus level did not response for adding phytase, while it increased due to citric acid supplementation. This variance may be related to the differences in supplementation levels of MP and experimental conditions.

Table (4): Effect of phytase and/or citric acid supplementation on plasma and tibia content of calcium and phosphorus.

\begin{tabular}{|c|c|c|c|c|c|c|c|c|c|c|}
\hline \multicolumn{3}{|l|}{ Treatments } & \multicolumn{2}{|c|}{$\begin{array}{l}\text { Blood calcium } \\
(\mathrm{mg} / \mathrm{dl})\end{array}$} & \multicolumn{2}{|c|}{$\begin{array}{l}\text { Blood phosphorus } \\
(\mathrm{mg} / \mathrm{dl})\end{array}$} & \multicolumn{2}{|c|}{$\begin{array}{l}\text { Tibia calcium } \\
\%\end{array}$} & \multicolumn{2}{|c|}{$\begin{array}{l}\text { Tibia phosphorus } \\
\%\end{array}$} \\
\hline \multirow[t]{2}{*}{ A.P \% } & \multirow{2}{*}{$\begin{array}{l}\mathrm{MP} \\
\mathrm{FTU} / \mathrm{kg}\end{array}$} & \multirow{2}{*}{$\begin{array}{l}\mathrm{CA} \\
\%\end{array}$} & $0-3$ & $0-5$ & $0-3$ & $0-5$ & $0-3$ & $0-5$ & $0-3$ & $0-5$ \\
\hline & & & $\mathrm{Wk}$ & wk & wk & $\mathrm{Wk}$ & Wk & wk & wk & wk \\
\hline $\mathrm{T} 1,0.45$ (PC) & - & - & 11.31 & 9.54 & $5.72^{c}$ & $5.54^{\mathrm{ab}}$ & $11.79^{\mathrm{bc}}$ & $11.81 \mathrm{a}^{\mathrm{b}}$ & $9.17^{b}$ & $6.01^{\mathrm{cd}}$ \\
\hline $\mathrm{T} 2,0.25(\mathrm{NC})$ & 0 & 0 & 10.31 & 10.03 & $5.32^{\mathrm{cd}}$ & $5.33^{\mathrm{bc}}$ & $15.97^{\mathrm{a}}$ & $12.68^{\mathrm{ab}}$ & $8.77^{\mathrm{bc}}$ & $6.71^{\mathrm{ab}}$ \\
\hline $\mathrm{T} 3,0.25$ & 0 & $1 \%$ & 10.72 & 9.67 & $5.61^{\mathrm{bc}}$ & $5.44^{\mathrm{bc}}$ & $12.91^{b c}$ & $12.21^{\mathrm{ab}}$ & $9.11^{b}$ & $6.28^{\mathrm{bc}}$ \\
\hline $\mathrm{T} 4,0.25$ & 0 & $2 \%$ & 11.67 & 9.61 & $6.11^{\mathrm{a}}$ & $5.03^{\mathrm{c}}$ & $11.58^{\mathrm{bc}}$ & $12.60^{\mathrm{ab}}$ & $8.72^{\mathrm{bc}}$ & $6.03^{\mathrm{cd}}$ \\
\hline $\mathrm{T} 5,0.25$ & 500 & 0 & 11.17 & 9.74 & $5.41^{\mathrm{bcd}}$ & $5.48^{\mathrm{abc}}$ & $12.40^{\mathrm{bc}}$ & $12.27^{\mathrm{ab}}$ & $9.09^{\mathrm{b}}$ & $6.87^{\mathrm{a}}$ \\
\hline T6, 0.25 & 500 & $1 \%$ & 11.08 & 9.45 & $5.10^{\mathrm{d}}$ & $5.50^{\mathrm{abc}}$ & $12.15^{\mathrm{bc}}$ & $13.17^{\mathrm{a}}$ & $8.99^{b}$ & $6.28^{\mathrm{bc}}$ \\
\hline $\mathrm{T} 7,0.25$ & 500 & $2 \%$ & 9.74 & 9.67 & $5.24^{\mathrm{d}}$ & $5.46^{\mathrm{abc}}$ & $12.20^{\mathrm{bc}}$ & $12.02^{\mathrm{ab}}$ & $9.08^{b}$ & $5.72^{\mathrm{d}}$ \\
\hline $\mathrm{T} 8,0.25$ & 1000 & 0 & 11.52 & 9.67 & $5.72^{b}$ & $5.64^{\mathrm{ab}}$ & $10.76^{\mathrm{c}}$ & $12.21^{\mathrm{ab}}$ & $8.21^{\mathrm{c}}$ & $6.11^{\mathrm{cd}}$ \\
\hline $\mathrm{T} 9,0.25$ & 1000 & $1 \%$ & 10.95 & 9.93 & $5.28^{\mathrm{dc}}$ & $5.94^{\mathrm{a}}$ & $13.17^{\mathrm{b}}$ & $11.25^{\mathrm{b}}$ & $10.20^{\mathrm{a}}$ & $6.03^{\mathrm{cd}}$ \\
\hline $\mathrm{T} 10,0.25$ & 1000 & $2 \%$ & 10.44 & 9.68 & $5.22^{\mathrm{d}}$ & $5.62^{\mathrm{ab}}$ & $12.66^{\mathrm{bc}}$ & $12.35^{\mathrm{ab}}$ & $9.01^{b}$ & $6.21^{\mathrm{c}}$ \\
\hline SEM & & & 0.65 & 0.217 & 0.116 & 0.153 & 0.68 & 0.43 & 0.207 & 0.15 \\
\hline \multicolumn{11}{|c|}{ Overall Means Phytase } \\
\hline 0.25 & 0 & - & 10.90 & 9.77 & $5.68^{a}$ & $5.26^{\mathrm{b}}$ & $13.49^{\mathrm{a}}$ & 12.49 & 8.87 & 6.34 \\
\hline 0.25 & 500 & - & 10.66 & 9.62 & $5.25^{\mathrm{b}}$ & $5.48^{b}$ & $12.25^{\mathrm{b}}$ & 12.91 & 9.06 & 6.29 \\
\hline 0.25 & 1000 & - & 10.97 & 9.76 & $5.41^{b}$ & $5.74^{\mathrm{a}}$ & $12.20^{\mathrm{b}}$ & 11.9 & 9.14 & 6.12 \\
\hline \multicolumn{11}{|c|}{ Overall Means Citric } \\
\hline 0.25 & - & 0 & 11.04 & 9.81 & 5.49 & 5.48 & 13.04 & 12.38 & $8.69^{b}$ & $6.56^{\mathrm{a}}$ \\
\hline 0.25 & - & $1 \%$ & 10.91 & 9.68 & 5.33 & 5.62 & 12.74 & 12.32 & $9.43^{\mathrm{a}}$ & $6.2^{\mathrm{b}}$ \\
\hline 0.25 & - & $2 \%$ & 10.61 & 9.65 & 5.52 & 5.37 & 12.15 & 12.21 & $8.94^{\mathrm{b}}$ & $5.99^{b}$ \\
\hline \multicolumn{3}{|c|}{ Interaction MP x CA } & NS & NS & 0.01 & NS & 0.006 & NS & 0.02 & 0.004 \\
\hline
\end{tabular}

A.V, available phosphorus; MP, microbial phytase; CA, citric acid; PC, positive control (0.45\% phosphorus); NC, negative control $(0.25 \%$ phosphorus)

$a, b, c, d$ Mean values within a column with no common superscript differ significantly from each other $(P<0.05)$. SEM, standard error of means; NS, not significant

The value \pm stander error 
Tibia calcium of chicks fed NC diet was higher than those fed PC diet or NC diet enriched with MP and/or CA; however, the differences lacked significance at the $5^{\text {th }}$ week of age. It can be suggested that low AP in NC diet stimulate releasing more phosphorus molecules from bone in a physiological attempt to keep blood calcium to phosphorus ratio constant. Releasing phosphorus molecule may relatively increase the ratio of calcium because of bone composed of tricalcium phosphate, in this concern the references are still absence. Brenes et al. (2003) noticed a significant increase in tibia calcium of broiler chicks due to feeding low AP diet $(2.5 \mathrm{~g} / \mathrm{kg}$ diet) illustrating that reduced dietary AP level increased plasma calcium which reflects on increasing calcium deposition in tibia.

Tibia content of phosphorus did not response clearly for adding MP or CA into NC diet. Hariharan \& Gangadevi (2015) did not find any significant differences in tibia content of phosphorus due to reducing dietary AP by $40 \%$ or by adding phytase or CA into low P diet. In contrary several studies indicated that, addition of MP and/or CA into low AP diet enhance phosphorus releasing from phytate complex which can reflect in enhancing phosphorus deposition in tibia bone (Brenes et al., 2003; Zobac et al., 2004; Sheikh et al., 2010).

\section{Nutrient Retention:}

Nitrogen and calcium retention of chicks fed NC were significantly $(\mathrm{P} \leq 0.05)$ lower than those fed PC diet or NC diets supplemented with 1000 FTU/kg MP and CA (Table 5). Zobac et al. (2004) observed an improvement in digestibility of nitrogen by adding lactic acid and phytase into low AP broiler diets. The improvement in protein utilization and retention by adding phytase may be related to its effect on reducing nitrogen-phytate complex, which inhibits protein digestion (Abd El-Hack et al., 2018). Furthermore, dietary organic acid reduces gastric $\mathrm{pH}$ and increases the rate of pepsinogen transformation to pepsin which promotes protein digestion and retention (Khodambashi Emami et al., 2013). Similarly, Manobhavan et al. (2016) noted an increase in calcium retention by adding phytase into low AP diet. They related this improvement in calcium retention to release more calcium molecules from phytate bonds by the action of phytase. Deepa et al. (2011) suggested that, low intestinal pH resulting from citric acid supplementation may increase solubility and absorption of calcium, as well, make phytic acid more protenized preventing formation of calcium phytate complex and increasing calcium retention. Phosphorus retention of chicks fed NC diet was significantly similar for those fed PC or NC diets supplemented with MP and/or CA (Table 5). It can be suggested that the birds have a greater ability to retain more phosphorus from $\mathrm{NC}$ diet, in a response for rising plasma phosphorus level. Therefore, the phosphorus retention $\%$ was not affected clearly because it calculated as percentage of total intake which is low in NC diet. Brenes et al. (2003) noted an enhancement in phosphorus retention of chicks fed low AP diets. In contrary, Deepa et al. (2011) reported that, phytase and citric acid supplementation improved phosphorus retention due to their beneficial effect on releasing more phosphorus molecules from phosphorus-phytate-complex.

\section{CONCLUSION AND APPLICATIONS}

1. Adding phytase enzyme at $500 \mathrm{FTU} / \mathrm{kg}$ level with $1 \%$ citric acid into NC diet was effectiveness to improve body weight and tibia bone characteristics as like as those fed PC diet or NC enriched with $1000 \mathrm{FTU} / \mathrm{kg}$ phytase enzyme.

2. This finding can be applied successfully in poultry formulation for increasing the utilization of phytate phosphorus, reducing the ratio of inorganic phosphorus and phytase supplementation units as well, controlling the pollution resulting from extra phosphorus excretion.

3. There is no clear effect of citric acid or phytase enzyme supplementation on plasma or tibia content of phosphorus. 
Table (5): Effect of phytase and/or citric acid supplementation on Calcium and phosphorus retention.

\begin{tabular}{|c|c|c|c|c|c|}
\hline \multicolumn{3}{|c|}{ Treatments } & \multirow{2}{*}{$\begin{array}{c}\text { Nitrogen } \\
\text { Retention } \%\end{array}$} & \multirow{2}{*}{$\begin{array}{r}\text { Calcium } \\
\text { Retention \% }\end{array}$} & \multirow{2}{*}{$\begin{array}{r}\text { Phosphors } \\
\text { Retention \% }\end{array}$} \\
\hline A.P \% & MP FTU/kg & $\mathrm{CA} \%$ & & & \\
\hline $\mathrm{T} 1,0.45(\mathrm{PC})$ & - & - & $65.53^{\mathrm{a}}$ & $55.33^{\mathrm{a}}$ & $53.30^{\mathrm{ab}}$ \\
\hline $\mathrm{T} 2,0.25(\mathrm{NC})$ & 0 & 0 & $58.60^{\mathrm{c}}$ & $47.93^{\mathrm{d}}$ & $54.30^{\mathrm{a}}$ \\
\hline $\mathrm{T} 3,0.25$ & 0 & $1 \%$ & $59.70^{\mathrm{bc}}$ & $50.57^{\text {bcd }}$ & $50.43^{\mathrm{ab}}$ \\
\hline $\mathrm{T} 4,0.25$ & 0 & $2 \%$ & $58.03^{\mathrm{c}}$ & $51.30^{\mathrm{bcd}}$ & $48.10^{\mathrm{b}}$ \\
\hline $\mathrm{T} 5,0.25$ & 500 & 0 & $60.13^{b c}$ & $48.80^{\mathrm{cd}}$ & $54.83^{\mathrm{a}}$ \\
\hline T6, 0.25 & 500 & $1 \%$ & $59.87^{\mathrm{bc}}$ & $53.80^{\mathrm{ab}}$ & $53.63^{\mathrm{ab}}$ \\
\hline $\mathrm{T} 7,0.25$ & 500 & $2 \%$ & $59.93^{\mathrm{bc}}$ & $48.73^{\mathrm{cd}}$ & $51.63^{\mathrm{ab}}$ \\
\hline $\mathrm{T} 8,0.25$ & 1000 & 0 & $63.53^{\mathrm{ab}}$ & $53.00^{\mathrm{ab}}$ & $49.33^{\mathrm{ab}}$ \\
\hline $\mathrm{T} 9,0.25$ & 1000 & $1 \%$ & $64.87^{\mathrm{a}}$ & $55.47^{\mathrm{a}}$ & $52.50^{\mathrm{ab}}$ \\
\hline $\mathrm{T} 10,0.25$ & 1000 & $2 \%$ & $63.67^{\mathrm{ab}}$ & $52.27^{\mathrm{abc}}$ & $50.63^{\mathrm{ab}}$ \\
\hline SEM & & & 1.23 & 1.17 & 1.70 \\
\hline \multicolumn{6}{|c|}{ Overall Means Phytase } \\
\hline 0.25 & 0 & - & $58.78^{\mathrm{b}}$ & $49.94^{\mathrm{b}}$ & 50.94 \\
\hline 0.25 & 500 & - & $59.98^{b}$ & $50.44^{\mathrm{b}}$ & 53.37 \\
\hline 0.25 & 1000 & - & $64.02^{\mathrm{a}}$ & $53.58^{\mathrm{a}}$ & 50.82 \\
\hline \multicolumn{6}{|l|}{ Overall Means Citric } \\
\hline 0.25 & - & 0 & 60.76 & $49.91^{\mathrm{b}}$ & 52.82 \\
\hline 0.25 & - & $1 \%$ & 61.48 & $53.28^{\mathrm{a}}$ & 52.19 \\
\hline 0.25 & - & $2 \%$ & 60.54 & $50.77^{\mathrm{b}}$ & 50.12 \\
\hline Interaction MP x C & & & NS & NS & NS \\
\hline
\end{tabular}

A.V, available phosphorus; $M P$, microbial phytase; $C A$, citric acid; $P C$, positive control ( $0.45 \%$ phosphorus); $N C$, negative control ( $0.25 \%$ phosphorus).

$a, b, c, d$ Mean values within a column with no common superscript differ significantly from each other $(P<0.05)$. SEM, standard error of means; NS, not significant.

The value \pm stander error.

\section{EFERENCES}

Abd El-Hack, M. E.; Alagawany, M.; Arif, M.; Emam, M.; Saeed, M.; Arain, A.; Farman, A.; Siyal, A.; Patra, A.; Elnesr, Sh. S.; and Khan, R. U. (2018). The uses of microbial phytase as a feed additive in poultry nutrition-A review. Ann. Anim. Sci., 18, 3: 639-658.

Ahmad, T.; Rasool, S.; Sarwar, M.; and Zia-ulHasan, A. U. (2000). Effect of microbial phytase produced from a fungus Aspergillus niger on bioavailability of phosphorus and calcium in broiler chickens. Anim. Feed Sci. Tech., 83, 2: 103-14.

AOAC (1990). Association of Official Analytical Chemists. Official Methods of Analysis, 15th edn. Washington, D.C., USA.

Aydin, A.; Pekel, A. Y.; Issa, G.; Demirel, G.; and Patterson, P. H. (2010). Effects of dietary copper, citric acid, and microbial phytase on digesta $\mathrm{pH}$ and ileal and carcass microbiota of broiler chickens fed a low available phosphorus diet. J. Appl. Poult. Res. 19: 422-431.

Boling, S. D.; Webel, M.; Mavromichalis, I.; Parson, C. M.; and Baker, D. H. (2000). The effects of citric acid on phytate-phosphorus utilization in young chicks and pigs. J. Anim. Sci. 78: 682-689. 
Brenes, A.; Viveros, A.; Arija, I.; Centeno, C.; Pizarro, I.; and Bravo, C. (2003). The effect of citric acid and microbial phytase on mineral utilization in broiler chicks. Anim. Feed. Sci. Tech. 110: 201-219.

Chung, T. K.; and Baker, D. H. (1990). Phosphorus utilization in chicks fed hydrated sodium aluminosilicate. J. Animal Sci. 68: 1992-1998.

Cowieson, A. J.; Hruby, M.; and M. Pierson, E. E. (2006). Evolving enzyme technology: impact on Commercial poultry nutrition. Nutr. Res. Rev. 19: 90-103.

Crenshaw, T. D.; Peo, E. R.; Lewis A. J.; and Moser, B. D. (1981). Bone strength as a trait for assessing mineralization in swine: A critical review of techniques. Anim. Sci. J. 53:827- 835.

Deepa, C.; Jeyanthi, G. P.; and Chandrasekaran, D. D. (2011). Effect of phytase and citric acid supplementation on the growth performance, phosphorus, calcium and nitrogen retention on broiler chicks fed with low level of available phosphorus. Asian J. Poult. Sci. 5: 28-34.

Duncan, D.B. (1955). Multiple range and multiple F test. Biometrics, 11:1-42.

Ebrahimnezhad, Y.; Shivazad, M.; Taherkhani, R.; and Nazeradl, K. (2008). Effects of citric acid and microbial phytase supplementation on performance and phytate phosphorus utilization in broiler chicks. J. Poult. Sci., 45.

El-Afifi, Sh. F. (2003). Comparative studies on garlic bulb, virginiamycin and citric acid as growth promoters in broiler chicks' diet. J. Agric. Sci. Mansoura. Univ., 283371 - 3381.

El-Afifi, Sh. F.; El-Medney, N. M.; and Attia, M. (2001). Effect of citric acid supplementation in broiler diets on performance and intestinal microflora. Egypt. Poult. Sci. 21: 491-505.

Hariharan, T.; and Gangadevi, P. (2015). Efficacy of Citric Acid and Microbial Phytase on the Tibial Characteristics, Tibial and Serum Mineral Concentrations in Broiler Chicken. Indian J. Anim. Res., 49, 3 : 328-332.

Ismail, A.; Bilal, T.; and Eseceli, H. (2011). The effect of organic acid, zeolite, or their combination on performance, some serum indices, and ileum $\mathrm{pH}$ values in broilers fed with different phosphorus levels. Turk. J. Vet. Anim. Sci. 35: 337-344.

Khodambashi Emami, N.; Zafari Naeini, S.; and Ruiz-Feria, C.A. (2013). Growth performance, digestibility, immune response and intestinal morphology of male broilers fed phosphorus deficient diets supplemented with microbial phytase and organic acids. Livest. Sci., Volume 157, Issues 2-3, $506-513$.

Luciana, P. N.; Rodrigues, P. B.; Meneghetti, C.; Bernardino, V. M. P.; Oliveira, D. H. D. H.; Saldanha, M. M.; Teixeira, L. V.; and Santos, L. M. (2016). Efficiency of microbial phytases in diets formulated with different calcium: phosphorus ratios supplied to broilers from 35 to 42 days of age. J. App. Ani. Res., 44, 446-453.

Manobhavan, M.; Elangovan,A. V.; Sridhar, M.; Shet, D.; Ajith,S.; Pal, D. T.; and Gowda, N. K. (2016). Effect of super dosing of phytase on growth performance, ileal digestibility and bone characteristics in broilers fed corn-soya-based diets. J. Anim. Phys. Anim. Nut. 100: 93-100.

Nourmohammadi, R.; Hosseini, S. M.; and Farhangfar, H. (2010). Effect of dietary acidification on some blood parameters and weekly performance of broiler chickens. J. Anim. Vet. Adv., 9: 3092-3097.

Nourmohammadi, R.; Hosseini, S. M.; Farhangfar, H.; and Bashtani, M. (2012). Effect of citric acid and microbial phytase enzyme on ileal digestibility of some nutrients in broiler chicks fed corn-soybean meal diets. Italian J. Anim. Sci. 11: 35-40.

NRC (1994). National Research Council. National Requirements of Poultry. 9th. Edn., National Academy Press, Washington, DC. ISBN: 13: 978-0-309-04892-7.

Redcliffe, J. S.; Zhang, Z.; and Kornegay, E. T. (1998). The Effect of microbial phytase, citric acid, and their interaction in a corn-soybean meal-based diet for weanling pigs. J. Animal Sci. 76: 1880-1886.

Sacakli, P.; Sehu,A.; Ergün, A.; Genc, B.; and Selcuk, Z. (2006). The effect of phytase and organic acid on growth performance, carcass yield and tibia ash in quails fed diets with low levels of non-phytate phosphorus. Asian-Aust. J. Anim. Sci. 19: 198-202.

SAS (1998). SAS User's Guide. SAS Inst. Inc. Cary, NC. 
Sheikh, A.; Banday, T.; Gulam, A. B.; Masood, S.; and Manzoor, R. (2010). Effect of dietary supplementation of organic acids on performance, intestinal histomorphology, and serum biochemistry of broiler chicken. Vet. Med. Int., (14): 1-7.

Soliman, N. K.; Al-Youssef, Y. M.; and Ganeem, N. B. (2012). Improving broiler performance and feed efficiency by adding organic acid to broiler diets. Egypt. J. Nut. and Feeds, 15: 393-404.

Sreeja, A.; Divya,S.; Jyotirmoy, G.; Vaibhav, B. A.; Karthik, B.; Dintaran, P. P.; and Arumbackam, V. E. (2018). Effect of immobilized fungal phytase on growth performance and bone traits of broilers fed with low dietary calcium and phosphorus. Vet. World, 11, 6: 758-764.

Talebi, E.; Zarei, A.; and Abolfathi, M. E. (2010). Influence of three different organic acids on broiler performance. Asian J. poult. Sci. 4, 1: 7-11.

Vieira, B. S.; Caramori Junior, J. G.; Oliveira, C.F.S.; and Correa, G.S.S. (2018). Combination of phytase and organic acid for broilers: role in mineral digestibility and phytic acid degradation. Worlds Poult. Sci. J., Vol. 74, 711-726.

Woyengo, T. A.; Slominski, B. A.; and Jones R. O. (2010). Growth performance and nutrient utilization of broiler chickens fed diets supplemented with phytase alone or in combination with citric acid and multicarbohydrase. Poult. Sci. 89:2221-2229.

Zhang, B.; and Coon, C. N. (1997). The relationship of various tibia bone measurements in hens. Poult Sci. 76, 12: 1698-701.

Zivkov, B. M.; Kova, E. M.; Mihaljev, Z.; Stojanov, I.; Kapetanov, M.; Stojanovi, D.; and Petrovi, J. (2012). The Effectiveness of Phytase in Broiler Diets in Improving Production Performances and Bone Features. Acta Vet. 62. 3: 297-311.

Zobac, P.; Kumprechi, L.; Suchy, P.; Strakova, E.; and Heger, J. (2004). Influence of L-lactic acid on the efficacy of microbial phytase in broiler chickens. Czech J. Anim. Sci., 49 (10): 436-443.

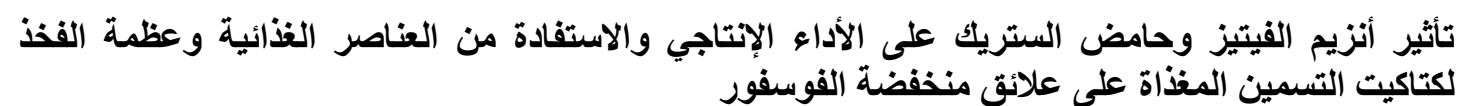

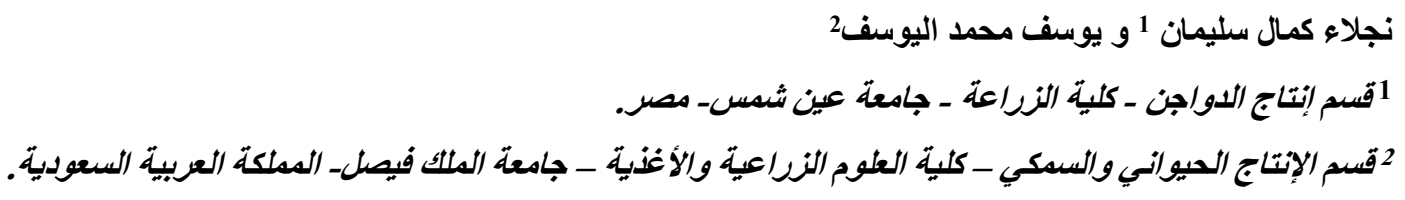

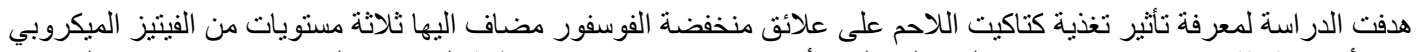

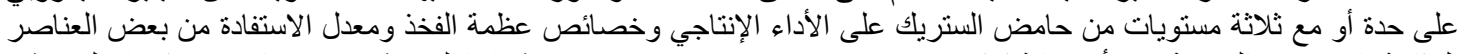

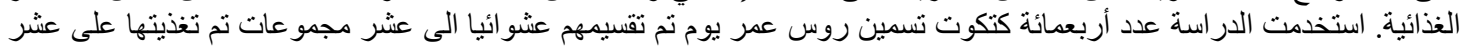

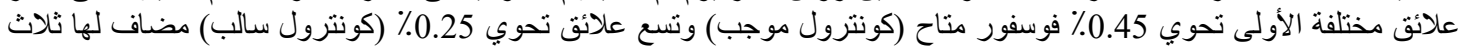

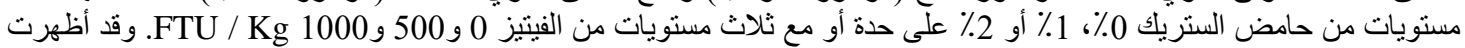

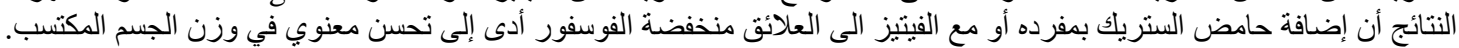

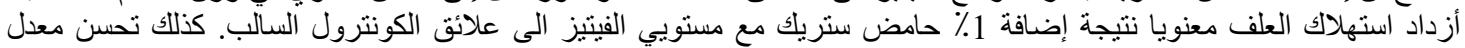

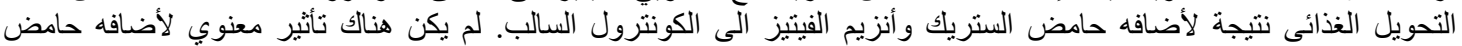

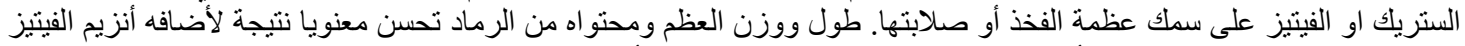

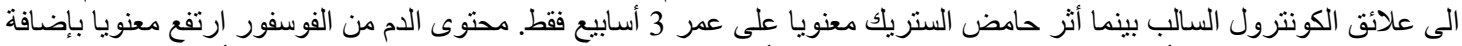

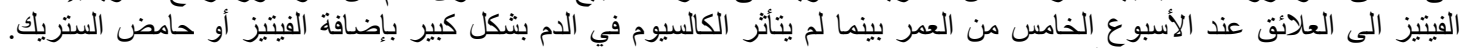

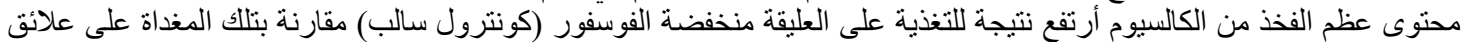

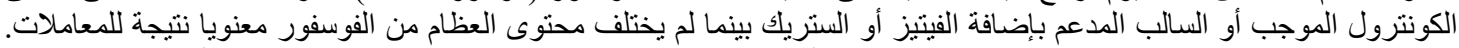

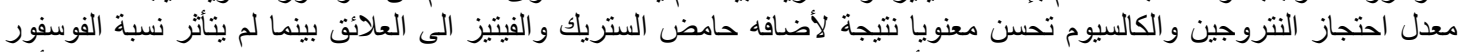

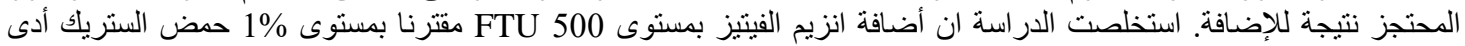

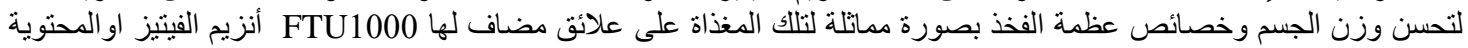
على مستوى كاف من الفوسفور. 